\title{
Relative bioavailability of pilocarpine from a novel ophthalmic delivery system and conventional eyedrop formulations
}

\author{
J A KELly, P D MOLYNeUX, S A SMITH, And S E SMITH
}

From the Division of Pharmacological Sciences and Toxicology, United Medical and Dental Schools of Guy's and St Thomas's Hospitals, London SE1 7EH

SUMmaRY Pupillary responses to pilocarpine following topical application in a novel ophthalmic delivery system (NODS) and a conventional eyedrop formulation have been compared in eight healthy subjects in a single dose crossover study. The magnitude of the miotic and light reflex responses to NODS 40,80 , and $170 \mu \mathrm{g}$ and to a single $2 \%$ Minims eyedrop (delivering $518 \mu \mathrm{g}$ ) were recorded by infrared television pupillography over periods of 24 hours. Dosage comparisons of drug responses were obtained by interpolation and yielded the equivalence of one eyedrop to $67 \pm 11 \mu \mathrm{g}$ pilocarpine from the NODS formulation. These findings indicate that in a comparison of total doses delivered pilocarpine has an approximately eight-fold greater bioavailability from NODS than from a conventional eyedrop formulation.

Pilocarpine is conventionally applied as eyedrops in aqueous solutions of various concentrations. Alternative technologies for drug delivery have been developed (solutions in castor oil, Ocuserts, and others), but these have not found wide clinical application. A novel ophthalmic delivery system (NODS) has recently been designed to deliver a precise amount of the drug to the eye. The medication is incorporated into a water-soluble polyvinyl alcohol (PVA) film which detaches from the rest of the device (a stiffened paper strip handle) when placed in the lower conjunctival sac (Fig. 1). The medicated film (length $4 \mathrm{~mm}$, width $6.3 \mathrm{~mm}$, thickness $20 \mu \mathrm{m}$, volume $0.5 \mu \mathrm{l}$ ) dissolves in the tear fluid releasing the medication. The overall length is 55 $\mathrm{mm}$, the whole device being of similar size to a Fluoret. The NODS contains no preservative and is sterilised by gamma radiation.

We report here a study in healthy volunteers designed to compare bioavailabilities from NODS and conventional eyedrop formulations.

\section{Material and methods}

Subjects. Eight healthy subjects (4 M, 4 F) aged 19 to 36 years took part in the study. None had a previous Correspondence to Professor S E Smith, Department of Clinical Pharmacology, St Thomas's Hospital, London SE1 7EH. history of cardiovascular, respiratory, or eye disease, and all had normal eyes, intraocular pressure (IOP), blood pressure, electrocardiogram (ECG), and haematological and biochemical profiles. One female subject was taking oral minocycline $50 \mathrm{mg}$ daily; there were no other drug treatments. Each gave written consent to participate, and the study was approved by the Ethics Committee of West Lambeth Health Authority.

Treatments and study design. Subjects attended on four occasions at the same time of day separated by at least seven days. No caffeinated drinks or smoking were permitted on experimental days. On each occasion one of four treatments, each containing pilocarpine nitrate, was administered in a single dose to one eye only in a complete balanced crossover design using two Latin squares. The four treatments were: NODS $40 \mu \mathrm{g}$, NODS $80 \mu \mathrm{g}$, NODS $170 \mu \mathrm{g}$,

STIFFENED HANDLE

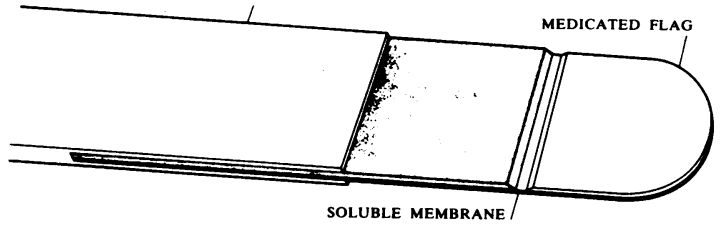

Fig. 1 NODS-a novel ophthalmic delivery system. 
and $2 \%$ aqueous eyedrops (a single drop delivered from Minims pilocarpine $2 \%$ ). The average weight of an eyedrop was independently recorded and calculated to deliver $518 \mu \mathrm{g}$ of pilocarpine nitrate.

Measurements. Before and at 15, 30, 45, 60, 75, and $90 \mathrm{~min}$, and at $2,3,4,6,9$, and $24 \mathrm{~h}$ after treatment, darkness pupil diameters and amplitudes of reflex responses to $0.5 \mathrm{~s} \mathrm{light}$ flashes were recorded from both eyes by infrared television pupillography as previously described. ${ }^{1}$ Drug effects on diameter and light reflex amplitude at each time were calculated as differences between treated and untreated eye. The total miotic response was calculated as the area under the curve from time 0 to $24 \mathrm{~h}$ by the trapezoid rule. Effects on the light reflex were calculated as percentage inhibition.

The subjects were questioned about local adverse effects of the formulations, stinging, foreign body sensation, and eye watering at 1 and 20 min after treatment and the results graded 0 (absent), 1 (mild), 2 (moderate), or 3 (severe).

Statistical analysis. The repeatability of the measurements of pupil diameter and light reflex amplitude was assessed from the pretreatment data by analysis of variance. The values, which were derived from the within-subject residual mean square, yielded coefficients of variation as follows: pupil diameter, $3 \cdot 6 \%$; light reflex amplitude $11 \cdot 1 \%$. The intraocular differences showed poor repeatability because of the low mean differences involved, but the standard deviations were in each case small by comparison with the changes induced by drug treatment.

In each subject graphs of the log dose pilocarpineresponse relationships for the NODS formulations were plotted and the equivalent dose for the eyedrop formulation calculated by interpolation. Mean values, standard errors of mean, and $95 \%$ confidence intervals for these estimates were calculated by standard methods.

\section{Results}

Pilocarpine constricted the pupil in all doses studied. The time course of this constriction is shown in Fig. 2. Peak responses occurred at median times of $0.75 \mathrm{~h}$ (NODS $40 \mu \mathrm{g}$ and $80 \mu \mathrm{g}$ ) and $0.5 \mathrm{~h}$ (NODS $170 \mu \mathrm{g}$ and eyedrops) without significant difference between them. The magnitude of the peak effects were ranked in order: NODS $170 \mu \mathrm{g}>$ NODS $80 \mu \mathrm{g}$ >eyedrops $>$ NODS $40 \mu \mathrm{g}$. Light reflexes were inhibited in parallel with miosis. Mean peak effects and area under the curve (AUC) are given in Table 1.

The dose-response relationship for pupillary constriction from NODS formulations was non-linear, the highest dose used $(170 \mu \mathrm{g})$ producing an effect

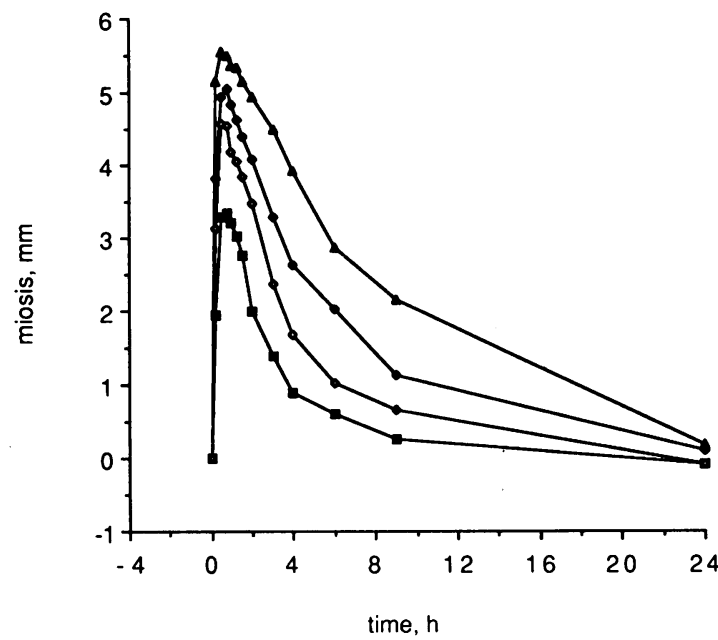

Fig. 2 Mean pupillary constriction responses to pilocarpine following application of NODS $40 \mu \mathrm{g}$ (ロ—- $), 80 \mu \mathrm{g}$ $(\longrightarrow)$, and $170 \mu \mathrm{g}(\mathbf{\Delta}-\mathbf{\Delta})$ and eyedrop formulations $(\diamond-\diamond)$.

close or equal to a maximum (Fig. 3). The dose of the eyedrop formulation was submaximal in its effect, and the calculated NODS equivalence was $67 \cdot 1$ (SEM 10.7) $\mu \mathrm{g}$ with $95 \%$ confidence intervals of 45.7 and $88.5 \mu \mathrm{g}$ (Table 1). The relationships for the total miosis (AUC) and for light reflex inhibition yielded almost identical figures. On the basis that one eyedrop delivered $518 \mu \mathrm{g}$ pilocarpine, these findings indicate in terms of total dosage an increase in relative bioavailability of the NODS formulation of $518 / 67 \cdot 1$ or $7 \cdot 7$-fold.

No serious side effects or adverse reactions were experienced. A number of subjects complained of local effects immediately following instillation of the NODS or eyedrops. Application of NODS induced a transient foreign body sensation in the eye on 22 of 24 occasions but eyedrops did so on only 1 of 8 occasions

Table 1 Pilocarpine-induced peak miosis, total response (area under the curve-AUC), and light reflex inhibition following administration as NODS and eyedrop formulations. Values expressed as mean (SEM)

\begin{tabular}{|c|c|c|c|}
\hline & $\begin{array}{l}\text { Peak miosis } \\
(\mathrm{mm})\end{array}$ & $\begin{array}{l}\text { AUC Miosis } \\
(m m h)\end{array}$ & $\begin{array}{l}\text { Light reflex } \\
\text { inhibition } \\
(\%)\end{array}$ \\
\hline NODS $40 \mu \mathrm{g}$ & $3.42(0.58)$ & $12 \cdot 23(2 \cdot 78)$ & $60 \cdot 3(8 \cdot 8)$ \\
\hline NODS $80 \mu \mathrm{g}$ & $5 \cdot 07(0.36)$ & $33.78(7.04)$ & $88 \cdot 2(5 \cdot 0)$ \\
\hline NODS $170 \mu \mathrm{g}$ & $5.67(0 \cdot 38)$ & $50 \cdot 70(7 \cdot 34)$ & $95 \cdot 0(1 \cdot 7)$ \\
\hline Eyedrops 2\% & $4.65(0.47)$ & $22 \cdot 07(4 \cdot 78)$ & $78 \cdot 4(4 \cdot 1)$ \\
\hline $\begin{array}{l}\text { NODS equivalent of } \\
\text { eyedrops }(\mu \mathrm{g})\end{array}$ & $67 \cdot 1(10 \cdot 7)$ & $65 \cdot 6(10 \cdot 8)$ & $68.5(18 \cdot 3)$ \\
\hline (95\% Confidence interval) & $45 \cdot 7-88 \cdot 5$ & $45 \cdot 1-87 \cdot 2$ & $31 \cdot 9-105 \cdot 1$ \\
\hline
\end{tabular}




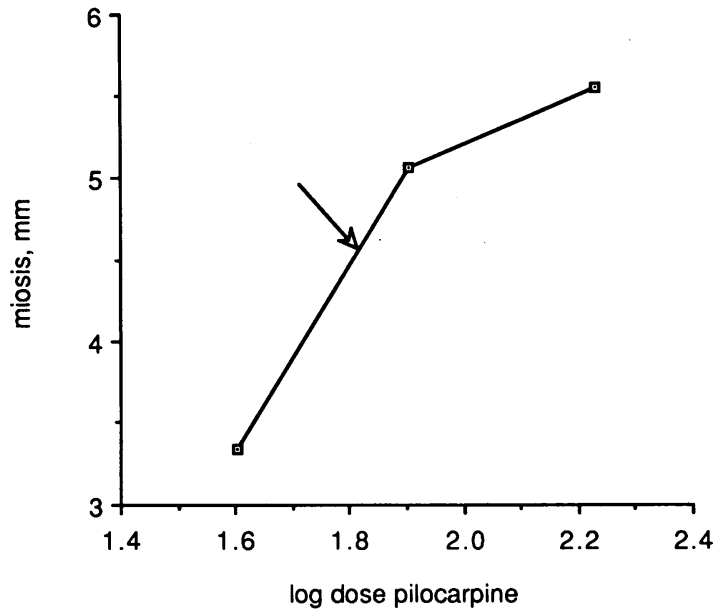

Fig. 3 Log dose/mean peak response relationships for pilocarpine following application of $\operatorname{NODS}(40,80$, and 170 $\mu g)$. The arrow indicates the mean peak response for the eyedrop formulation.

$\left(\chi^{2}=19 \cdot 014, p<0.001\right)$. In only one subject on one occasion did mild discomfort persist for $20 \mathrm{~min}$. Stinging and eye watering occurred equally with the two types of formulation.

Intense miosis in response to the treatments was associated with eye or brow ache on the treated side during the first two hours after instillation. Headache, severe enough to require medication with paracetamol, occurred in two subjects thereafter. These responses were dose related and not associated with a particular formulation.

\section{Discussion}

The quantity of drug applied to the eye from conventional eyedrops is necessarily high because of the low bioavailability from this route. At least $80 \%$ of the volume of an administered eyedrop drains rapidly through the nasolacrimal duct, allowing for subsequent systemic absorption. ${ }^{2}$ The use of polyvinyl alcohol as a vehicle for ophthalmic drugs, first described in Russia in $1964,{ }^{2}$ greatly improves bioavailability. ${ }^{3}$ The results of the present study indicate that pilocarpine in NODS can be applied at 8 times smaller dosage than in eyedrops with a proportionally reduced risk to the patient of systemic side effects. Clinically pilocarpine eyedrops rarely produce such effects when used in chronic simple glaucoma, but the high dose and frequency of treatment in acute glaucoma may cause the classical signs of muscarinic receptor overstimulation. The resulting symptoms include increased salivation, nausea, vomiting, diarrhoea, bronchospasm, and pulmonary oedema. $^{45}$

Many different ophthalmic drugs could be delivered in NODS vehicles. The serious systemic side effects from beta-blocker therapy in chronic simple glaucoma in particular might thereby be reduced.

The volunteers in this study tolerated NODS reasonably well and the ease of application was comparable to eyedrops. The transient foreign body sensation might discourage some patients. Polyvinyl alcohol is thought to be non-irritant to the eye, ${ }^{h}$ and the small film in NODS has not been associated with adverse reactions in other studies.

We thank Smith and Nephew Pharmaceuticals for a gift of the materials and for financial support. SAS is supported by the Research Endowment Committee, Special Trustees of West Lambeth Health District.

\section{References}

1 Smith SA, Smith SE, Lazare R. An increased effect of pilocarpine on the pupil by application of the drug in oil. Br J Ophthalmol 1978; 62: 314-7.

2 Shell JW. Pharmacokinetics of topically applied ophthalmic drugs. Surv Ophthalmol 1982; 26: 207-18.

3 Grosz I v, Takacsi Nagy G v. Ein Losungsmittel mit protrahierter Wirkung für die Ophthalmologie. Arzneimittelforschung 1967; 17: 1213-5.

4 Epstein E, Kaufman I. Systemic pilocarpine toxicity from overdosage in treatment of an attack of angle-closure glaucoma. $\mathrm{Am} \mathrm{J}$ Ophthalmol 1965; 59: 109-10.

5 Greco JJ, Kelman CD. Systemic pilocarpine toxicity in the treatment of angle closure glaucoma. Ann Ophthalmol 1973; 5: 57-9.

6 Krishina N, Mitchell B. Polyvinyl alcohol as an ophthalmic vehicle: effect on ocular structures. Am J Ophthalmol 1965: 59: 860-4.

Accepted for publication 4 August 1988. 